ARTICLE

DOI: $10.1057 /$ s41599-018-0126-4

\title{
Analysing the role of virtualisation and visualisation on interdisciplinary knowledge exchange in stem cell research processes
}

\author{
Neil Stephens (10 1, Imtiaz Khan² \& Rachel Errington ${ }^{3}$
}

\begin{abstract}
Interdisciplinary work is an increasingly frequent and important aspect of scientific research. However, successful knowledge exchange and collaboration between experts is itself a challenging activity with both technical and social components that require consideration. Here, this article analyses the cultural factors involved in interdisciplinary research, specifically in the context of a software programme designed to improve knowledge exchange. The authors undertook an ethnographic study to understand the impact of virtualisation and visualisation on an interdisciplinary research team, working together to develop novel imaging technologies for investigating stem cells. In this discovery-research environment the challenge was to determine the socio-technical effect of an in-house software tool called ProtocolNavigator, which provided a virtual laboratory environment for the team to simulate and map their 'real-life laboratory' activities. This display, together with activity icons and a timeline, could be visually analysed and transferred between multiinstitutional researchers in a non-narrative approach. The aim of the work reported here is to provide the first detailed analysis of how software of this type is used in practice, and to analyse this through a robust social science-based perspective. Subsequently this study examines the effectiveness of this virtual laboratory environment on enhancing communication and understanding using a theoretical framework drawing upon three insights from the Sociology of Expertise and Experience: (i) interactional and contributory expertise, (ii) knowledge exchange and interpretative flexibility, and (iii) trading zones. The framework was extended by introducing the notion of material, concept and practice trade at these trading zones with a demonstration of how interactive visualisation of interconnected trading routes can empower interdisciplinary work. The addition of Foucault's classic account of power found evidence of a panoptical effect due to the augmented visibility of each other's work. In summary, the article offers a theoretical framework together with novel analysis to determine the influence of introducing tools and approaches designed to enhance interdisciplinary working-particularly in the context of knowledge sharing and trust.
\end{abstract}

\footnotetext{
${ }^{1}$ Social and Political Sciences, Brunel University London, Kingston Lane, Middlesex UB8 3PH England, UK. ${ }^{2}$ School of Technologies, Cardiff Metropolitan University, Cardiff CF5 2YB Wales, UK. ${ }^{3}$ School of Medicine, Cardiff University, Tenovus, Heath Park, Cardiff CF14 4XN Wales, UK. These authors contributed equally: Neil Stephens, Imtiaz Khan. Correspondence and requests for materials should be addressed to N.S. (email: Neil.Stephens@Brunel.ac.uk)
} 


\section{Introduction}

here is a long tradition of empirically analysing scientific knowledge production using the methodological and theoretical approaches of the social sciences (Knorr, 1977, Latour and Woolgar, 1985, Lynch, 1985, Traweek, 1988, Jasanoff et al., 1995, Alač, 2011, Vertesi, 2015). The current study contributes to this literature by analysing interdisciplinary scientific knowledge work using qualitative methods. Specifically, we look at a site centred on, but not limited to, biological stem cell science and the use of a specific software package designed to aid knowledge exchange.

Recent decades have witnessed the shift within biology from a singular laboratory-based science towards a data-intensive interdisciplinary science. This has led to an exponential increase in the volume and variety of biological data as well as substantial growth of the number and diversity of interdisciplinary collaborations. With this have come new ways of organising and valuing computational biological work, seen most clearly in the emergence of bioinformatics as a leading space for conducting this work (Lewis et al., 2016, Bartlett et al., 2018). Consortia or large scale projects are usually based on highly organised collaborations, undertaking scientific activities in a manufacturing-style environment with standard operating procedures and a great degree of automation, but often little room for creativity. Therefore, the data or biological products generated from such collaborations can be of high quality but rarely generate new findings or ideas (Witt, 2008).

This contrasts with long-tail scientific work; the many smaller projects that if collated would produce more work than the smaller number of large scale projects (Wallis et al., 2013). These long-tail collaborations are usually organically grown or shaped by emergent ideas and available funding streams. Creative processes carried out in this discovery-like environment are the basis for new ideas and transformative science. The continued and unstructured mixing of expertise from 'wet' laboratory biology and 'dry' laboratory numerical research creates a 'moist zone'; an interdisciplinary landscape that remains characterised by difficult communication between scientists with distinct expertise (Penders et al., 2008). Communication within the interdisciplinary landscape of long-tail projects often stalls due to the lack of shared experience, discipline specific languages and jargons, and other cultural barriers. Within biomedical and data science communities this is sometimes called the 'human factor' (Hartswood et al., 2012), although here we argue this is better thought of as 'cultural factors'.

This study employs key ideas from sociology and Science and Technology Studies to better understand the challenge of these cultural factors in data science. We apply this to the empirical case-study of our in-house and open source software ProtocolNavigator; a virtual laboratory environment that enables emulation of real-life laboratory activities and automatic depiction of experimental design as an interactive map (Khan et al., 2014). The aim is to demonstrate how social science has a theoretically robust framework for analysing the impact of virtualisation and visualisation on interdisciplinary communication, especially in the context of knowledge sharing and trust.

The current study has been undertaken by an interdisciplinary team consisting of a sociologist (Stephens), a data scientist (Khan), and a cell biologist (Errington). Following Edwards et al. (2017), we argue interdisciplinary engagements of this sort are mutually beneficial to both individuals and disciplines as ideas and analysis are shared. The study aims to make discrete contributions to each field. First, it contributes to the social analysis of science by applying an established theoretical framework within this space-the Sociology of Experience and Expertise (SEE) - and augmenting it by adding new categories (material, concept, and practice trades) while embedding it within an analysis of surveillance and power. Second, it contributes to interdisciplinary laboratory work by articulating and providing a worked example of how the language of SEE can be used to understand and facilitate the knowledge exchange between people from different disciplinary backgrounds, and in doing so making explicit particular issues, and the reasons for those issues, that may otherwise be misunderstood or unacknowledged. Third, it contributes to data science by providing a novel and theoretically rich empirical analysis of the use of a particular software application designed to facilitate interdisciplinary exchange, which we use to develop critically engaged insight to challenges for the field as a whole. We begin our discussion by detailing more about the context of data science, and the role of virtualisation within it.

\section{Data science and virtualisation}

Data science is playing an increasing role in developing technical solutions that address the management, knowledge discovery (Burgun and Bodenreider, 2008) and analysis of data. E-science solutions like grid or cloud computing enable distributed research and data sharing, while ICT solutions like computer mediated communication (CMC) technologies (e.g., email, videoconference) through to social network-like collaborative environments (e.g., myExperiment) (De Roure et al., 2009, 2010) are providing support for better communication and cooperation that are widely utilised by scientists from both large and long-tail scientific projects. Despite these technological advancements, laboratory visits and staff rotation remains the prevalent route to gain experience, where social interaction in real world and faceto-face communication provides the basis for shared learning. However, as the number and geographical distance between collaborative groups are increasing and the funding is decreasing, it is often difficult to provide this effective but expensive interaction for gaining experience (Romano et al., 2011).

Virtualisation of real world scenarios in which users from different geographical locations can interact and experience each other's activities and skills via collaboration, competition, and creativity has demonstrable impact on sharing experience (Herbert et al., 2013, Snowdon et al., 2001). Our in-house software ProtocolNavigator utilises a virtualisation approach through which researchers work to emulate laboratory activities on a virtual bench, particularly the sequential activities of stem cell culture; maintenance, assessment and measurement. ProtocolNavigator is designed to emulate all the stem cell experimental activities and thus automatically draw the design of the experiment as an interactive map with activity icons on a timeline. Multiple researchers are able to contribute their activities and cumulatively generate a map intended to reflect the whole experimental design with an augmented visibility of each user's contribution. Traversing through the links within the depicted experimental design provides audit-trail information (who, where, when, what) about the individual's work, that should aid the end user of the data, or samples, to better understand the broader process. As we will show, visualising and understanding each other's work in this virtual ecosystem also provides a basis for both collective intelligence and surveillance.

In this study, we analysed these social interactions and perceptions through an interview and ethnographic study. We deployed ProtocolNavigator within a team that consisted of biologists, physicists, engineers and chemists, a cast of 20 people. They worked to produce (i) new techniques to drive stem cell differentiation through controlling the biophysical microenvironment of the biology, (developing new ways to control how stem cells grow), (ii) new non-linear microscopy modes to measure and monitor stem cells (essentially new types of readouts 
from a microscope that give the user new ways to measure stem cells), and (iii) new biological barcodes that can be added to cells to enable the tracking of specific stem cell lineages over time (allowing new ways of following what happens to stem cells as they grow). The team is characterised by bringing together scientists from epistemologically and culturally distinct disciplines to work on developing new, creative technologies and ways of understanding. As such, we describe the group as an interdisciplinary' team because they seek to provide a solution to a problem that cannot be adequately dealt with by one discipline alone (Klein and Newell, 1998). This stands in contrast to a multidisciplinary team that would work together but not seek to integrate their insights. Being a team, their work is a 'collaborative' or 'collective' interdisciplinarity, as opposed to the 'individual interdisciplinarity' of the single researcher who seeks to master two disciplines (Calvert, 2010, Lewis and Bartlett, 2013). Subsequently, our work delivers an analysis of team dynamics and interaction, in part digitally-mediated, among a diverse group of researchers addressing shared goals.

\section{Methodology}

As noted above, the authorship team of this study includes a social scientist (Stephens), a data scientist (Khan), and a cell biologist (Errington). Khan and Errington have developed the ProtocolNavigator software and collaborated with Stephens in designing the analysis of ProtocolNavigator reported here. The methodological approach that was adopted to understand users' experiences of ProtocolNavigator is squarely based on the longstanding social science approaches within the field of Science and Technology Studies: the empirical analysis of scientists' practice with a focus on the role of cultural aspects in the development and use of knowledge (Jasanoff et al., 1995, Felt et al., 2017).

In this study, we apply qualitative methods, including interviews, to understand how the interdisciplinary team of scientists worked together on a 4 -year research project. The team used, tested, and informed the developmental life-cycle of ProtocolNavigator in an iterative manner. In particular a concerted eight week trial was undertaken, during which the manipulations and measurements were made on cellular samples by these interdisciplinary and multi-institutional team members as their process was recorded through ProtocolNavigator. We analysed both this specific short term trial and also the broader 4-year project as part of an ethnographic study of interdisciplinarity in practice. Twenty-nine semi-structured interviews were conducted by Stephens over this 4 -year period with team members from across the range of expertise. Interviews lasted between one and three hours and were recorded and transcribed. Interviewees were asked about the challenges of working in an interdisciplinary context and about their expectations and practical experiences with ProtocolNavigator.

Ethnographic observations were also conducted and recorded in fieldnotes by Stephens at the three-monthly team meetings and during laboratory visits over the 4 -year period. Khan and Errington were also present at the majority of these meetings, although they did not produce fieldnotes. These day-long threemonthly team meetings in particular were key moments for data collection as the team members communicated their progress and negotiated challenges in conducting interdisciplinary work. Stephens is an experienced ethnographer who has conducted multiple ethnographies of scientific practice in other contexts (Stephens et al., 2011, Stephens et al., 2013a, Stephens and Lewis, 2017).

The project was approved by Cardiff University School of Social Sciences research ethics committee. As part of these ethical assurances participants and interviewees are given personal anonymity, so the accounts and interview quotations presented here do not identify the individuals involved, as is standard bestpractice in qualitative and ethnographic work. All team members were observed and approached for an interview by email, with 17 people interviewed, eight more than once, and two members not agreeing to be interviewed for undisclosed reasons. Interviews were held in the interviewees' office or a private seminar room with no one else present.

Interview transcripts and fieldnotes were analysed thematically deploying a coding framework that included situating and demographic features (e.g., disciplinary background, professional experience), generic modes of social engagement (e.g., trust relationships, face-to-face contact), and themes specific to the theoretical framework (e.g., contributory expertise, tacit knowledge). The relevance of the SEE framework was realised during the early stages of fieldwork, allowing its use in analysis as data collection was ongoing.

Due to the anonymity agreements made with interviewees, all direct analysis of the raw data was conducted by Stephens, with subsequent analysis by all authors conducted through ongoing dialogue and exchanges of text based upon a limited subset of anonymous data and reflection upon ethnographic experience. In what follows next we articulate the theoretical sociological ideas that underpin our analysis.

\section{An analytical framework for understanding interdisciplinarity} There are five components to our theoretical framework that effectively inform our understanding of how interdisciplinarity works and the challenges this poses for a virtualisation approach. The first three are taken from the SEE. These are: (i) interactional and contributory expertise, (ii) tacit knowledge, explicit knowledge and interpretative flexibility, and (iii) trading zones. The fourth builds upon SEE to study (iv) trust and social distance, and the fifth is a classic sociological account of $(\mathrm{v})$ power and surveillance. In combination all inform our understanding of the nature of interdisciplinary research and impact of virtualisation and visualisation. We follow this articulation of our approach with a discussion of multiple critiques and adaptions of the SEE framework and locate ourselves within them. However, we note our use of SEE stays closer to the original articulation found in Collins and Evans (2002, 2007), as augmented with our own conceptual additions.

Interactional and contributory expertise. Our starting point with the SEE framework is the distinction between interactional and contributory expertise. Contributory expertise is "what you need to do an activity with competence", and it stands in contrast to interactional expertise, which is "the ability to master the language of a specialist domain in the absence of practical competence" (Collins and Evans, 2007). Both are attained, and used, during interaction and involvement within scientific communities and both include a large component of specialist tacit knowledge. The team studied is full of contributory experts: the biologists are contributory experts in stem cell biology and tissue culture. Just as the engineers are contributory experts in rheology, they know the relationships between deformations and stress in liquids and soft solids (including cells), and they are able to acquire data using combined rheometric, imaging and molecular simulation studies techniques, further, they publish peer-reviewed papers accepted within the rheology community.

At the same time, like many interdisciplinary projects, it is also a situation where interactional expertise is being developed. The cell biologists start to learn some of the technical language of rheology, they can ask informed questions about the research, and understand how the findings about stress measurements on 
cells relate to their own contributory expertise in cell biology. Subsequently they can discuss, and discuss with increasing technical competence, but they cannot do, and thus they begin to develop what SEE terms interactional expertise.

Interactional expertise, especially in esoteric sciences, is not easily attained. It takes time and can only be achieved through immersion within a community of experts. Interactional expertise cannot be replaced by reading technical descriptions alone. Information based only upon reading-termed Primary Source Knowledge-is insufficient because it lacks the focus of our next theme from SEE: specialist tacit knowledge (Collins, 2010a, b) and experience.

Tacit knowledge, explicit knowledge and interpretative flexibility. The main purpose, and a core challenge, for virtualisation and hence ProtocolNavigator-like software is to make a process explicit and thus facilitate the building of knowledge about how stem cells have been cultured, to the team and eventually a wider peer-group. As such, it relates directly to a central notion within SEE, the relationship between tacit and explicit knowledge. According to Collins (2010a, b), explicit knowledge is knowledge that can be elucidated, that is written down or transferred by the use of strings, meaning patterns of information, in the right circumstances. This is in contrast to tacit knowledge, understood as "that which has not or cannot be made explicit" (Collins, 2010b). Knowledge may remain tacit because of the restricted ways people relate to each other, the restrictions of physical capabilities, or the complicated nature of social systems. Tacit knowledge can fall into two types: specialist and ubiquitous. Specialist is the skill of a trained laser physicist who calibrates and validates a complicated optical system, while 'ubiquitous' includes normal language use (it is something most people have learnt but still requires skill to perform competently). To read scientific papers-i.e., to gain Primary Source Knowledge-rests on the ubiquitous tacit knowledge of language comprehension and library and internet use. As noted, it lacks the immersion or experience within a community required to gain interactional and contributory expertise.

Tacit knowledge, including specialist tacit knowledge, can be divided into three forms. The first, somatic tacit knowledge, is 'knowledge stored in the muscles, nerve pathways, and synaptic connections' (Collins, 2010b), it is the craft skills and judgements of skilled practice. The second, relational tacit knowledge, is knowledge that could be made explicit, but is not (for good social reasons), with diverse reasons including lack of trust in the learner, or a lack of realisation that the learner needs to know (either because the knower assumes the learner already knows it, or the knower does something through taken-for-granted habit and does not realise its significance in accomplishing the desired result). As Collins notes, it is impossible to resolve this kind of problem simply by telling you 'everything' you might need to know because 'everything' is an open-ended category' (Collins, 2010b). The third type of specialist tacit knowledge, (called collective tacit knowledge), is knowledge that belongs to a social collective, and changes as driven by the community; it has no authoritative source.

Stem cell tissue culture for research and also next-generation cell therapies requires the tissue culture of cells, systematically expanded under laboratory conditions, meeting standards of robust validation and good laboratory practice. However, like any scientific discipline at the cutting-edge, this is a site of specialist tacit knowledge (Stephens et al., 2011). It is not uncommon for tissue engineers to talk of the craft-like skills typical of passaging and differentiating cell colonies; this is a clear example of somatic tacit knowledge. Practical skills are learnt through observing other people and through extensive training. Long tail cell biologists recognise their in-house procedures as idiosyncratic, in recognition of the multiple non-standardised approaches employed. As we discuss later, trust also plays an important role in making knowledge explicit, as knowledge holders choose whom to provide information to, and knowledge seekers determine whose knowledge ownership claims to believe (Collins, 2007, ReyesGalindo, 2014, Stephens et al., 2011).

Making the tacit explicit is further complicated by the related concept of interpretative flexibility. In short, it means that any information can be interpreted in multiple ways (Collins, 1992). Typically, when groups of people agree on an interpretation of any information, or indeed a standardisation, it is a product of a broader shared framework of meaning and conventions that close down alternative interpretations or practical processes.

Trading zones. Trading zones describe spaces in which scientists with different languages (expertises) come together to try and exchange knowledge and expertise. The term entered the sociology of knowledge when Galison developed the metaphor drawn from economic trades, such as goods, between people from completely different backgrounds, who are conducting trade potentially even in the absence of a shared language. It is used to understand how scientists manage to communicate when they do not fully understand each other's disciplines (1996). These trading zones can result in the formation of new languages, known as (in order of increasing complexity) jargons, pidgins, and creoles.

Embedding Galison's work within the SEE framework, Collins et al. (2007) introduce two key dimensions that strengthen our understanding of trading zones: a homogeneity-heterogeneity dimension and a coercion-collaboration dimension. The homogeneity-heterogeneity dimension describes how much the group members have in common in terms of shared languages, practices and tacit knowledge. A team of ProtocolNavigator users who are all stem cell biologists would have high homogeneity, while the interdisciplinary team in this study with a diverse range of contributory expertise have high heterogeneity. The second dimension-coercion-collaboration-captures the extent to which power is used to enforce interdisciplinary exchange. Groups that mutually come together have high collaboration, whereas those for which interdisciplinary work occurs through 'encouragement' or institutional force experience coercion. The team we studied can be thought of as a collaboration with coercive elements, as while the senior members chose to opt-in they did so in a context of a basic technology project and funding structure that encourages this interdisciplinary work.

Collins et al. (2007) cross the homogeneity-heterogeneity and coercion-collaboration dimensions in a diagram to identify four different types of trading zones: interlanguage, fractionated, subversive, and enforced. Two of these trading zones are relevant to our empirical case, the Fractionated Trading Zone, with high levels of collaborations but (or perhaps despite) high levels of heterogeneity, and the Interlanguage Trading Zone, with high collaboration and high homogeneity, perhaps because the traders have a shared background or perhaps due to the emergence of a new jargon. Figure 1 shows the two axis and four trading zones in a format based upon Collins, Evans and Gorman's original.

We have found it productive to further expand this framework to identify three types of trade that can happen in trading zones: (i) material, (ii) concept, and (iii) practice. Material trades involve trading physical (including electronic) phenomena such as tissues, recorded data sets, or software, and requires no additional expertise beyond knowing the traded material is appropriate for your task. Concept trades involve trading ideas and understandings: they can involve both explicit and tacit knowledge, and 


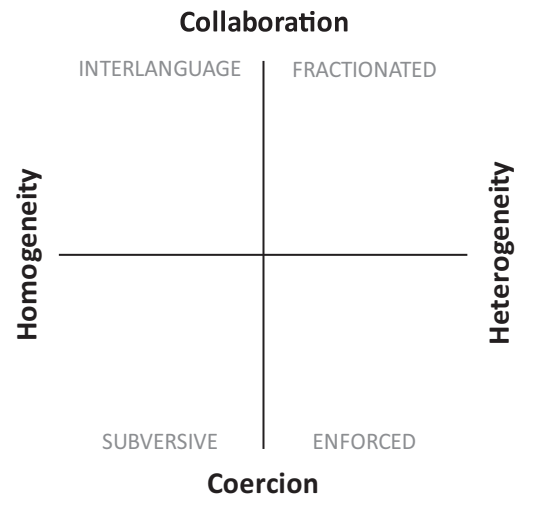

Fig. 1 Trading zones. The four trading zones (interlanguage/fractionated/ subversive and enforced) are shown divided across the collaboration/ coercion and homogeneity/heterogeneity axis (diagram is an adapted and simplified version of that from Collins et al., 2007, with the original charting six collaboration types across the same axis)

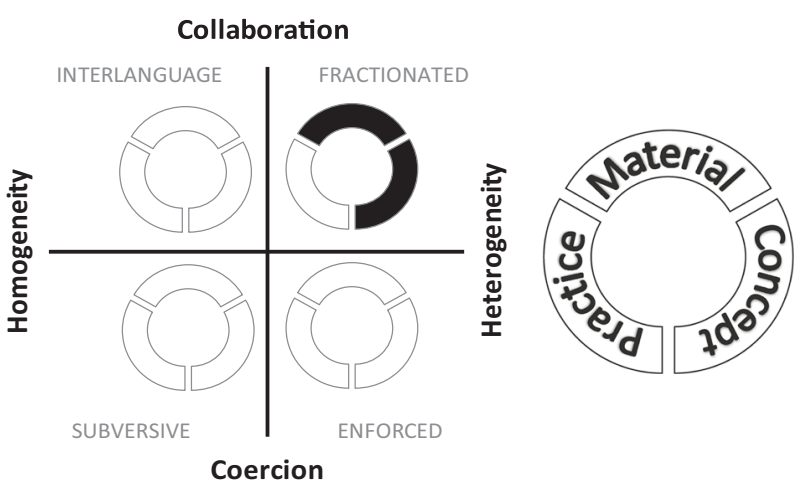

Fig. 2 Trading zones and material, concept and practice trades. The trading zone diagram augmented with the material/concept/practice doughnut to indicate the type of trade (here, a material and concept trade in the fractionated trading zone)

are the basis of exchanging interactional expertise. Trades of practice involve trading skills sets and capabilities, meaning the capacity to do something, and are the basis of exchanging contributory expertise. To clarify an important element of how we think about this, one stem cell biologist giving another stem cell biologist some standard stem cells should be understood as just a material trade. While of course both stem cell biologists have contributory expertise in stem cell biology, they do not need to exchange the skill set of how to culture stem cells because both already have that skill set. The relevance of this distinction for visualisation software will be made explicit later. Below we augment a version of Collins, Evans and Gorman's original depiction with our new categories in Fig. 2.

Figure 2 integrates our new categories of material, concept and practice trades into Collins et al. (2007) depiction of the four trading zone types (as seen in its original form in Fig. 1). The right hand side contains the legend that shows a labelled threepart doughnut divided into material, practice and concept segments. We include this to show how we represent a single trade. In the main section of the diagram we see a trade embedded with the trading zone diagram. The figure depicts two key elements. First, this is a trade within a fractionated trading zone (i.e., between people with high levels of collaboration and heterogeneous expertise), which is evident because the trade doughnut is in the top right quadrant. Secondly, this trade is a material and concept (but not practice) trade, which is evident because the two segments of the doughnut labelled as material and concept in the legend are shaded in black while the practice segment is unshaded. Later in this study we will be using these visualisations to demark what of type trade occurred among the group we studied during a test protocol.

Trust and social distance. Trust is a key component of any social interaction, whether mediated by software or not. Reyes-Galindo (2014) develops a model of trust in the sciences that combines Collins and Evans' SEE account with classic sociological accounts of trust (Giddens, 1990, 1991). Importantly for all systems like ProtocolNavigator, Reyes-Galindo introduces the notion of 'social distance' to capture the extent to which scientists have shared knowledge, cultural, and geographic proximity. He develops a typology of how different levels of social difference are associated with different types of trust, and allow different types of knowledge to be exchanged.

When scientists work closely together, with constant contact and minimal social distance, you find 'inter-personal trust' (see also Collins, 2001, MacKenzie, 2001, and for stem cell specific examples, Lewis and Atkinson, 2011, Morrison, 2017, and Stephens et al., 2011). Trust relationships of this type involve what Giddens (1990) calls 'facework commitments'; the labour of being with the people you want to trust, and want to trust you, and demonstrating competence and reliability through everyday interaction. Reyes-Galindo argues inter-personal trust supports the transmission of explicit, collective, and somatic tacit knowledge.

When scientists come into contact less frequently, with medium social distance, scientific knowledge exchange occurs through either 'trust-by-proxy' or 'institutional trust'. Trust-byproxy involves a chain of inter-personal connection via proxy. It is a type called 'referred trust' (Giddens, 1991). Institutional trust involves trusting someone based upon their professional affiliations and track record, without necessarily meeting in person. It is a type of 'systemic trust' (Giddens, 1991). In the context of scientists from different disciplines, both referred trust and systemic trust draw upon what Collins and Evans (2007) call 'meta-expertises': skills and cultural knowledge that allow individuals to understand the context of other knowledge cultures through acquaintance with their own. Examples range from the practical to the cultural and include knowledge of aseptic technique, or experimental design, or peer-review, or promotion trajectories. This is a referred social knowledge about what implies good scientific practice, and relies upon cultural proximity and sharing a social context (e.g., academia, biological sciences, etc). The closer the cultural space, the greater the trust meta-expertise can support. Reyes-Galindo argues these trust relations support the exchange of explicit and relational tacit knowledge, but not somatic or collective.

Finally, when scientists have very little physical or cultural contact, and maximal social distance, the greatest trust they can have is what Reyes-Galindo terms 'suspension of doubt'. This involves a trust granted to knowledge without formal basis. An example could be a novel 'black-boxed' technology whereby a scientist uses a machine or some software without knowledge of its workings or a social basis for that trust. This is a limited type of trust, that Reyes-Galindo argues only supports the transition of explicit knowledge to the exclusion of all tacit forms. He presents his typology in a table, adapted in Table 1.

Power and surveillance. In this last theoretical section, we draw upon the classic sociological and philosophical account of Foucault $(1975,1978)$ on power and its relationship to surveillance. As noted above, Collins et al. (2007) expansion of Galison's 
Table 1 Table of the relationships between trust, social distance and knowledge (adapted from Reyes-Galindo, 2014)

Relation between trust, social distance and type of knowledge that can be exchanged

\begin{tabular}{lll}
\hline Social distance & Type of trust & Characteristic type of knowledge exchanged \\
\hline Minimal & Inter-personal trust & Collective, somatic tacit knowledge, explicit technical skills \\
Medium & Trust-by-proxy / institutional trust & $\begin{array}{l}\text { Relational tacit knowledge/explicit technical knowledge, meta-expertises, referred social } \\
\text { judgement } \\
\text { Maximal }\end{array}$ \\
& Suspension of doubt & Explicit knowledge
\end{tabular}

notion of Trading Zones introduced a coercion-collaboration dimension (as well as a homogeneity-heterogeneity dimension) that introduces the notion of power to the SEE framework. Here, we want to expand that understanding of power by integrating Foucault's understanding of power not as a top-down force but as something which is diffuse and everywhere in every social relationship. Individuals are active in producing their own position within a power structure through self-disciplining, even when they are the dominated group. Foucault illustrated this with the example of Jeremy Benthams' Panopticon; a prison design in which prisoners' cells are arranged in a large circle all facing inwards with full visibility, and a central watch tower. Importantly there are direct sight lines from all cells to all other cells, and from the watch tower to all cells, but, the prisoners cannot see into the watch tower to know whether it is occupied by a guard or not. In this context of mutual-surveillance, (i) the prisoners self-discipline themselves even in the absence of a watching guard, and (ii) have their identities as prisoners reinforced by this surveillance.

We are not the first to consider Panoptical effects in the digital realm, with other examples including Bucher (2012) on Facebook, Lupton (2012) on M-health, and van Nuenen (2016) on multiplayer online computer games. Such work exists within a debate in Surveillance Studies over the continued relevance of Foucauldian analysis in this context. Some, most notably Haggerty and Ericson (2000), urge for a shift towards a framework that better captures the non-geographical, diffuse, and less top-down form of the digital world, in their case through the extension of Deleuze and Guattari (1987) in the concept of the 'surveillant assemblage'. Essentially the critique here would be that contemporary and digital realms skew the centralised and unequal power relationship between observer (watching guard) and observed (prisoner) inherent to the Panopticon, because digital environments can allow individuals to both observe and be observed, and are not linked to a single location (see also Bauman and Lyon 2013). Countering this, Caluya (2010) argues Foucault's thought is more continuous with Deleuze and Guarrati's than many Surveillance Studies scholars give it credit, and that Foucault's notion of power and the gaze ably captures this multiplicity. We will return to the importance of this for our empirical case-study, and SEE more widely, in the following empirical and discussion sections of this study.

Critiques and developments of the SEE framework. There are a number of published critiques of the SEE framework, which generally fall into two categories. The first category consists of those publications that critically engage with SEE's deployment to issues in science policy and politics. Since the outset, one intended use of SEE was to provide a social-demarcation criteria for judging which voices should be included as expert perspectives in controversial public policy decisions. This type of critique has limited relevance to our deployment of the framework, but we direct the interested reader to Wynne (2003), Rip (2003), Jasanoff (2003), Fischer (2011), Epstein (2011), Owens (2011), Forsyth
(2011), Jennings (2011) and Sismondo (2017) for examples of this type of critique, and Collins and Evans (2003), Collins et al. (2010), Collins et al. (2011) and Collins et al. (2017) for examples of SEE proponents defending their case.

The second category of critique with the SEE framework engages with Collins and Evans' (2007) definitions of interactional and contributory expertise, and the work these concepts can achieve. One example is Ribeiro and Lima (2016), who seek to discredit the notion of interactional expertise. They do so by challenging a set of claims made within SEE, examples including the claims that interactional expertise can be acquired through linguistic socialisation alone; known as the 'minimal embodiment thesis'. They argue empirical work shows people attaining interactional expertise do so through 'physical contiguity', i.e., typically spending time close to the practices being learnt, and that any learning of a language requires a form of embodiment. In a rebuttal paper, Collins and Evans (2016) argue Ribeiro and Lima miss the philosophical point being made. Collins and Evans (2016) recognise physical proximity is indeed a more efficient way to gain expertise (and thus how it is usually done in practice), but argue the minimal embodiment thesis is about whether it is the only way. In terms of our data-set, the participants are frequently physically co-located, so this is not an issue we can contribute to empirically, however, we will assess the significance of this debate to our ProtocolNavigator case in the discussion.

Another critique of SEE is found in Atkinson and Morriss' (2017) evaluation of the utility of the approach as a methodological aid to inform ethnographers of what type of competency they require to conduct ethnographic work. Atkinson and Morriss argue SEE is not best suited for this purpose, claiming SEE's notion of 'ubiquitous expertise'-intended to capture the competent conduct of everyday social tasks-misleadingly represents the everyday as expertise, and that, echoing Ribeiro and Lima (2016), the SEE framework privileges talk over participation. In rebuttal, Collins and Evans (2017) argue Atkinson and Morriss' dismissal of ubiquitous expertise mistakenly replaces a notion of expertise as the possession of socially relevant knowledge with a notion of expertise of status. Of more relevance to our study, and furthering the critical engagement with the Ribeiro and Lima (2015) argument, Collins and Evans look to dispel the distinction between language and practice by arguing that language speaking is a practice that is overwhelmingly premised upon social interaction and embodied and physical participation in a social realm.

In work seeking to improve the SEE framework, both Plaisance and Kennedy (2014) and Goddiksen (2014), identify two definitions of interactional expertise within Collins and Evan's work. Both identify an earlier definition that Plaisance and Kennedy argue is focused upon what is needed to allow someone to "interact interestingly" with a contributory expert (Collins and Evans, 2002, p. 254), and that Goddiksen argues relates to the capacity to speak the language of the domain. For both, the second definition of interactional expertise relates to whether an interactional expert can pass what Collins and Evans (2007) call 
the 'imitation game', a Turing test-like experiment in which an expert judge asks questions of two players-one another contributory expert and the other someone trying to be an interactional expert-to see if the judge can tell them apart through text-based question-and-answer exchanges. Both Plaisance and Kennedy and Goddiksen advocate for the earlier, nonimitation game definition, which is the one we employ as articulated earlier. Goddiksen, like us, is specifically interested in using SEE to study interdisciplinarity, and makes the argument that using a broader definition of interactional expertise (to, for example, include university students) furthers this agenda, while also arguing that text-based primary source knowledge alone can inform low level interactional expertise, a point that stands in opposition to Ribeiro and Lima's (2016) insistence that physical contiguity is a necessity, and also countered by Reyes-Galindo and Duarte (2015) who reassert Collins and Evans original account that immersion is essential. Plaisance and Kennedy's (2014) interest in the earlier definition is to expand those included as interactional experts to further democratise science, as part of what they call a pluralistic approach to interactional expertise. In this account they urge analysts to explore who interactional experts are, why they became experts, and how they use their expertise. Aiding this, Plaisance and Kennedy (2014) identify four general sets of motivations and the types of interactional experts associated with them: 'learners', 'challenges', 'facilitators', and-the category capturing the participants in our study-'collaborators'.

In a paper responding to both Plaisance and Kennedy (2014) and Goddiksen (2014), Collins and Evans (2015) highlight the risks of overly broadening the definition of interactional expertise to include students or political activists who are not immersed in the community of scientific experts. Specifically, to Collins and Evans, this weakens the utility of the SEE framework to intervene in the science policy issues discussed above and improve technological decision-making in the public domain. Subsequently, while our focus upon the earlier, non-imitation game definition of interactional expertise is aligned with Plaisance and Kennedy (2014) and Goddiksen (2014), our work does not seek to expand the definition to include broader sets of people, as our ethnographic work is exclusively within a scientific team who are not engaged in controversial public decision-making. Thus, our usage of this earlier definition is not subject to the critique of Plaisance and Kennedy (2014) and Goddiksen (2014) from Collins and Evans (2015). In our case, our usage of the nonimitation game definition is because our empirical setting is of contributory experts in one domain beginning the process of attaining interactional expertise in multiple other domains. This given, they are not close to attaining the maximal interactional expertise that Collins and Evans (2015) say the imitation game is designed to identify, and thus it provides limited insight here. We return to the perspectives of Plaisance and Kennedy and Goddiksen in relation to Collins and Evan's critique in our discussion.

Finally, we suggest that our work, in some regards, is itself a critique of the existing SEE framework, in arguing that SEE fails adequately to deal with power and identity. While the coercion/ collaboration axis within the trading zone raises power as an issue, we argue this can and should be expanded, as we do here through our engagement with Foucauldian thought. We expand upon this in the empirical and discussion sections of the work. Given this work to improve the conceptualisation of power within SEE, and our introduction of material, concept, and practice trades in trading zones, we argue SEE is an adequate and able framework for analysing data on interdisciplinary work (as also argued in Gorman, 2002). We now turn to this empirical work.

\section{Deploying the theoretical framework to understand virtualisation-mediated interdisciplinarity}

In the following sections, we draw upon our social science empirical work with the team developing non-invasive imaging techniques to fingerprint stem cell differentiation (Stephens et al., 2013b, Downes et al., 2011), including the challenges of their project and how they relate to ProtocolNavigator. We analyse these findings with the theoretical ideas discussed above.

The trading zones of the team depicted through the experimental undertaking. During the interviews the team members frequently expressed positive attitudes about the concepts and vision for software such as ProtocolNavigator. The accounts identify multiple benefits: (i) data sharing between groups, (ii) moving paper lab book based auditing into an e-environment, (iii) big data processing across experiments, (iv) reproduction of experimental practice by other people and/or groups, and (iv) oversight and management. The team noted that to maximise these benefits it needed to be time efficient and intuitive.

The post-doctoral team (the actual day-to-day users) also expressed support for the long-term vision of ProtocolNavigator. Although their periods of usage were typically short and rarely extended over the 8 continuous weeks of use during the beta phase of the ProtcolNavigator development life-cycle, the sociological analysis on team member interaction provided important insights and explanation about the challenges of ProtocolNavigator-like software development. One of these periods of use involved a stem cell protocol that was fully documented in ProtocolNavigator by the team. Figure 3 shows this experimental map and includes our social analysis.

The upper section of Fig. 3, labelled 'Experimental Map' shows the ProtocolNavigator output from the trial experiment, mapping the experimental protocol with activity icons aligned with the timeline at the top. This map component is exactly the computer output that the team were working with and will be discussing in interview in the following sections. For ease of interpretation we have added four highlighted time points where the trading of cell samples was carried out between different expertise groups. These trading zones are enclosed by bars numbered 1-4, and the corresponding trading zone quadrant at the bottom section of Fig. 3, labelled 'Social Layer' show our social analysis of these trades, specifically, what type of trade it is (material, practice or concept) and which trading zone it occurred in (interlanguage, fractionated, subversive, or enforced). In all, $0 \mathrm{~h}$ to $168 \mathrm{~h}$ represented BIO1, in which the cells were seeded, incubated and manipulated until they could be transferred into six metrology tracks. The cells then transitioned into BIO2, with further incubation, further medium added/exchanged, and finally placed onto a microscope platform and image data acquired. We call this transition Trade 1 , and shown in the trading zone diagram that this trade involved only the movement of material (the cell samples) within an interlanguage trading zone between cell biology contributory experts (as depicted by the single shaded area at the top of the trade doughnut). Trade 2, at $648 \mathrm{~h}$, is quite different, as here the cells were passed from a biologist to a rheological engineer who initiates the ENG1 process. The diagram shows that this trade is in the fractionated trading zone as it is between people with different expertise. It is a trade of material (the cell samples), concepts (understanding the cells) and, importantly, practice (the capacity to keep the cells alive), as depicted by the three shaded areas in the trade doughnut in the top right fractionated trading zone. The fractionated Trade 2 was essential to retain the cell's viability during the rheological experiments. As we discuss later, exchanging practice is the most difficult of trades. In contrast Trade 3 at $649 \mathrm{~h}$, from biology to 


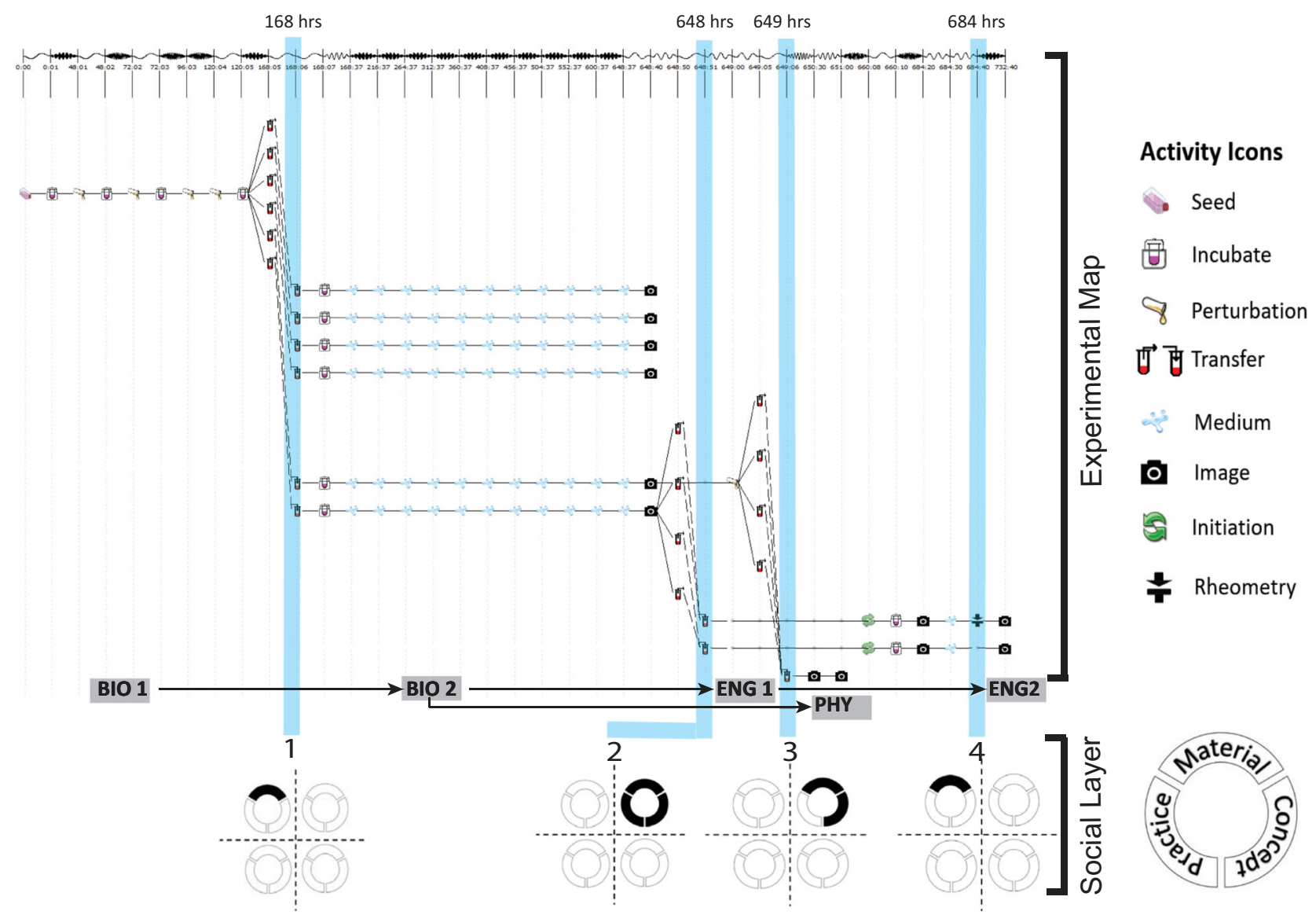

Fig. 3 Social analysis of an interdisciplinary stem cell experiment. The top section shows a screenshot of the ProtocolNavigator output, overlaid and linked to the bottom section with the trading zone social analysis of the experiment

physics, involves only material (the cell samples) and concepts (understanding the cells) as the cells did not need to be alive for the Coherent Anti-Stokes Raman Scattering (CARS) microscopy (Lu et al., 2015) undertaken by physicist (PHY). Later, we explore what 'understanding the cells' can mean for a physicist. Trade 4 at $684 \mathrm{~h}$ is once again a trade of material within an interlanguage trading zone, this time within engineering (ENG2), as the engineer conducts the rheological component on the cells they had successfully kept alive. Again, we will return to this trade later.

Expertise, trust and trading zones within the team. Working relationships within fractionated trading zones build upon a combination of inter-personal trust, referred trust, the emergence of a pidgin language, and the facework commitments of spending time together, as evident in this account from a senior stem cell biologist:

'Now the [rheologists], I've known them for a while. We've been on a number of joint projects with them. Probably about 10 years ago they came over to see us. They were talking about their things, we were talking about our things, and none of us understood each other. But we do now. We've met and we have some middle ground-they don't go too technical, we don't go too technical-and we can understand what we're talking about... And you know, in terms of what we want to do, they're the experts in the country, without any shadow of a doubt.'

The interviewees' trust in the rheology colleague's capabilities, as the nationally leading group, is premised not upon her/his own expertise in rheology (as she/he acknowledges that is limited), but instead on her/his meta-expertise of recognising the social demarcations of leading scientific practice. This trust was foundational to the working of the group in general, and the success of Trade 2 in Fig. 3.

As noted above, Trade 2, from biologist to rheologist, was the most challenging as the rheologist needed to maintain the viability of the cell culture away from the home or origin of the samples, meaning they required practical skills from another expertise as well as concepts and the material cells themselves. In practice they did this through attaining limited specialist tacit knowledge. To support Trade 2 a biologist provided a step-bystep account of how to culture cells specifically for the engineer, and felt $s /$ he had provided a clear account of all the steps. In practice the engineer was unable to successfully conduct/replicate these experiments, and could only resolve the situation through a face-to-face meeting with the cell biologist during which the engineer penned down notes and extra information on the original step-by-step guide. This significantly increased the quantity of text-explicating the taken-for-granted specialist tacit knowledge- until it made sense to the engineer. To address this issue in ProtocolNavigator a set of textual notes or audio-video accounts of individual activities could be embedded in the experimental map. This could help to improve enculturation in the tacit knowledge of each other's discipline.

Getting 'enough' explicit knowledge during the ProtocolNavigator trial. The impact of a fractionated trading zone with heterogeneous expertise was also evident during Trade 3 from biologist to physicist, as is clear in the following account 
from a physicist commenting on whether they understood the ProtocolNavigator report in Fig. 3 recording the cell biology:

'I would say yes [I understood the ProtocolNavigator output], I don't know why [the cell biologist] was doing that, but I could understand what s/he was doing. So, okay, in this particular moment s/he's using a particular chemical, or s/he's harvesting the cells and s/he's plating them in a different plating set-up, but why s/he was doing that, no I couldn't understand. Of course I have the general idea because s/he wrote part of the text in our maths paper because we were using her/his stats. Okay, I can read and see, okay s/he's treating the cell in a different way now to force it now, so the cells would be happy to develop in a different site, because you treat them in such way-no, I don't understand, I don't know, I just accept [her/his expertise]'.

This very revealing extract demonstrates some of the tensions of knowledge transfer in a fractionated trading zone. The ProtocolNavigator output made enough of the stem cell preparation and practice explicit for the physicist to articulate the basic categories of action undertaken (using chemicals, harvesting cells), but not the rationale for why these actions were pursued. This suggests only a limited form of interactional expertise, and there is no evidence of the somatic tacit knowledge transfer that would support the contributory expertise of being able to actually do cell culturing work. It suggests that users look for just enough explicit knowledge to let them get on with what they want to do. However, this just enough is clearly framed by an inter-personal trust relationship from working together to 'accept' her/his expertise.

We explore this theme further in another quotation from the same physicist, this time about their work recording data collected on their CARS-microscopy through the ProtocolNavigator interface:

'I would say [the use of ProtocolNavigator with CARSmicroscopy] was mostly successful. I also understand that [the software engineer] didn't want to add all the different information that I was discussing with him about the CARS-microscopy because obviously the CARSmicroscopy is a particular set-up... it was kind of compromised, we were adding some different filters so that we'll still give the idea of the different way to use the CARS microscope... we were still using the general microscope set-up list, but we were adding some additional fields so that it can be more similar to a CARS microscope'.

Here, the physicist is responding to the developmental state of the software that did not have a fully functional model of CARSmicroscopy in it. While instances like this are typical during software development, it again shows users thinking through what is enough knowledge. As a reverse of the previous quote, here the physicist suggests enough information was provided to allow others to have an understanding of the CARS-microscopy set-up, but not enough to inform a skilled practitioner on how to set-up it up with enough detail to repeat the work. The interviewee captures this enough-ness through the notion of similarity that allows the CARS/ProtocolNavigator encoding to be 'mostly successful' but also 'compromised'. Both these trades are examples of conceptual trades, as the experts exchanged understanding. However, as is clear in the interview quotations, both trades involved only limited understanding.

Virtualisation, power and identity in ProtocolNavigator-like systems. Recording and visualisation in a collaborative environment means ProtocolNavigator becomes a site of mutualsurveillance. As noted previously, using Foucault's (1975) notion of the panopticon, sites of surveillance are linked to power, and the associated self-disciplining and resistance. This is evident in an interview quote from a senior team member that articulates ProtocolNavigator's potential:

'as a PI [I like] how useful it could be because you do get access to all that data. At the minute it's in lab books, so you can bring them in, you could go through all the lab books, but that's tedious, that means I've got to involve [the postdocs who conducted the research]. If it's on a central system somewhere, I can delve into an experiment, I can follow through the results much more easily as PI'

Here, we can see how ProtocolNavigator becomes a site of surveillance in which existing power relationships are recast. The interviewee describes how the 'tedious' surveillance of post-docs can be simplified, and conducted without the post-doctoral scientists' input, allowing the PI to 'delve' and 'follow' experimental work from their desk space. The potential for surveillance was also recognised by a post-doctoral scientist, although their response to it is quite different:

'I don't necessarily want [senior members of staff] to be able to track absolutely everything I do... If you were to start being so closely monitored, I think a lot of people would start to feel bad about not hitting their targets and become very stressed, and there'll be a lot of pressure there'.

This 'pressure' is an articulation of Foucault's disciplinary power as ProtocolNavigator users self-regulate in line with the broader power structures they experience. In practice the PI may not want to check-up on their post-docs' progress, or indeed they may never even actually check the ProtocolNavigator software, but the post-doctoral scientists themselves can still experience and be complicit in this formation of power, just as the prisoner in the Panotpticon is complicit even though they never know if they are being watched. While the roles of PI and watch guard are different in many respects, ProtocolNavigator-like software are panoptic, and subsequently the visibility facilitated by ProtocolNavigator-like software may shape the laboratory practices they are intended to represent.

In a fractionated trading zone, with multiple disciplines, the potential visibility afforded by ProtocolNavigator-like software impacts identity as well as power. The response of users is not only framed by being visible, but how they are made visible (and accordingly how their identity is represented). In the previous example, the post-doctoral scientist experiences being made accountable for their actions through a heightened level of visibility. In contrast, in the next example, an engineer articulates dissatisfaction with limited opportunities for visibility offered to them and their work in ProtocolNavigator:

'I also felt it was set-up, as it was always going to be, from a cell biology perspective and that a lot of what we do in a rheology experiment, if you include it in ProtocolNavigator, it just looks like one little box with nothing to back it up. What ProtocolNavigator doesn't allow you to do is include the six weeks' worth of work previously, which allows you to carry out that one experiment and know that it's relevant. So you know, I was very aware that you'd have this cell biology map of experiments that spanned for weeks and weeks and weeks, and in there you'd have us with just a little tiny block in there that didn't do justice to the amount of work that went into that block... there are many experiments that need to be associated with that one block'. 
This comment evidences the relationship between virtualisation, surveillance, and identity, and the unintended consequences this can have. The engineer above explains that the icon in Trade 4 of the Fig. 3 virtualisation map did not give sufficient representation to their labour and their expertise. This is particularly felt in the context of a fractionated trading zone as other viewers of the ProtocolNavigator output lack the interactional expertise in rheology to necessarily recognise the work required. In contrast to the previous account, the dissatisfaction here is that the structure of the software does not allow them the opportunity to display their labour and their knowledge.

\section{Discussion}

The discussion is divided into two sections. The first reconsiders the SEE framework and its critiques in regard to our empirical setting. The second articulates findings, learning points, and issues for further consideration in relation to ProtocolNavigatorlike tools.

Reconsidering SEE. The SEE framework has been developed with multiple utilities in mind, with a key focus being a model for discriminating between which experts should be involved in technological decision-making in publically contested cases. Our usage of SEE follows Gorman's (2002) and Goddiksen's (2014) in that we use it to better understand interdisciplinary research practice. Our framework draws heavily upon the various publications of Collins and Evans, and the related work of ReyesGalindo (2014) on trust. We also outlined a set of critiques and discussions of SEE, and we return to these debates in light of our empirical work.

One such critique of the SEE framework came from Ribeiro and Lima (2016), who offer the notion of 'physical contiguity' to capture how time spent close to the practice of the desired expertise is essential to gaining interactional expertise. Our empirical work does not allow us to comment on whether physical contiguity is necessary, but our empirical work does confirm Collins and Evan's (2016) argument that it is beneficial. Similar to Collins and Evan's (2017) response to Atkinson and Morriss (2017), our case-study demonstrates that speaking the language of this specialist domain is closely linked to sensemaking around the embodied and tacit practices of cell culturing.

These debates about proximity and embodiment do have relevance for ProtocolNavigator-like systems. While the specific case-study presented here was of an interdisciplinary group who did physically meet regularly, there is a longer term aspiration for ProtocolNavigator to be used internationally and in much more geographically dislocated contexts, potentially among scientists who never have personally met each other. On one level, this would provide a practical context in which to explore both Ribeiro and Lima's (2016) argument that physical contiguity is necessary for interactional expertise, and Goddiksen's (2014) contrary argument that low level interactional expertise can be achieved through reading text alone (primary source knowledge). This is because potentially in these contexts the team could be communicating through the ProtocolNavigator user interface alone. However, this possibility also leads us to consider wider issues about the 'who' and the 'what' of physical contiguity, and how this relates to the specifics of the team environment. For example, we would anticipate two contributory experts in cell biology would be able to share familiar cell culturing practice via ProtocolNavigator even if they personally had not met, as they have existing experience of the domain of cell biology (an interlanguage trade). However, this becomes more complex when the information being traded is innovative, potentially involving novel tacit knowledge about the physical handing of difficult to manipulate cell lines. This would be further complicated if the trade occurred between scientists from different disciplines, depending upon what interactional expertise they already held. The final complication is what level of understanding the scientists deemed was 'enough' for their purposes, and whether their purpose was to gain an understanding of the practices described, to recreate, or to modify them. The focus on motivation and relationship recalls Plaisance and Kennedy's (2014) argument for articulating situated details of this kind, although we cut short from employing their fuller pluralist approach and expanding the cohort of experts. We will return to what our consideration of motivation means for ProtocolNavigator-like systems in the following section.

The current study has gone beyond simply employing the SEE framework, and has expanded its context of use and its theoretical components. We have expanded its context of use by using it to closely inspect a specific ethnographic example of interdisciplinary work, and the role of computational mediation within that. This stands in contrast to the more frequent use of the framework in more explicitly political domains, such as vaccination controversies (Boyce, 2007a, b) or AIDs denialism (Weinel, 2007), or through bespoke methodological approaches such as imitation games (Collins et al., 2006).

We have expanded the SEE framework in two ways. First, we introduced the notions of material, conceptual, and practice trades, we demonstrated their significance to interdisciplinary working, and related them to the existing concepts of primary source knowledge, interactional, and contributory expertises. Second, we significantly expanded the analytical reach of the work on power within the existing trading zones framework by connecting it to Foucault's (1975) classic notion of power. This has enabled us to recognise ProtocolNavigator as a site of power and surveillance, and to allow the SEE framework to engage with the micro-operation of power within a scientific research team. Doing so made explicit the role of visualisation and virtualisation in reshaping social relationships, potentially in unanticipated ways. When introducing these ideas we noted the argument found most clearly in Haggerty and Ericson (2000) that studies of surveillance should move beyond Foucauldian analysis, and instead engage with theoretical ideas from Deleuze and Guattari (1987), specifically through Haggerty and Ericson's own conceptualisation of the 'surveillant assemblage' to better capture the decentralised and unequal power relationships of digital environments. It is indeed the case that ProtocolNavigator allows all users to view the inputted material of all other users. However, in our case (and potentinally many others), it is the post-doctoral researchers conducting the laboratory work whose efforts are more frequently recorded in ProtocolNavigator, as the managerial work of more senior figures operating at a step away from the physical manipulation of the cells is not recorded in ProtocolNavigator-like systems. Subsequently, the powerdynamic afforded by the differing levels of visibility within ProtocolNavigator-like systems continue to support panopticonism. Additionally, our interview data makes clear that the users' experience reflects this account. Subsequently, following Caluya (2010), we consider the Foucauldian analysis an able and appropriate framework for our case, and a valuable augmentation of the SEE framework that further extends its engagement with notions of power.

Reconsidering ProtocolNavigator. There is a strong recognition within the data science community that the successful implementation of software is shaped by the 'human factor' (Hartswood et al., 2012). However, we argue the community lacks a sociologically rigorous framework through which to articulate 
and understand this. Here, we have described one possible approach to address this gap: the Sociology of Expertise and Experience (SEE) combined with Foucault's account of surveillance. This theoretical framework provides a set of core concepts that allows both data scientists, and those engaged in interdisciplinary work, to better express the challenges they face. The framework deployed here helps us understand how groups of people establish shared practices as they work together. As such they are cultural, which is why we suggest the 'human factor' is better understood as 'cultural factors' as they extend beyond the individual and into the full richness of a group social experience.

We also stress the importance for data scientists of empirical work to explore the perceptions and practices of users and developers of software. Producing successful software requires collating the right expertise. We argue that in the development life-cycle of any software the theoretical and empirical aspect of social science needs to be integrated in order to deliver meaningful solutions. Importantly, as exemplified here, this empirical work needs to be informed by, and inform, a robust theoretical model. In our case the empirical work has been interviews and observations with an interdisciplinary team using ProtocolNavigator. We close by articulating five key learning points that we have taken from this exercise.

The challenge of knowledge exchange. Our work highlights just how hard it is to know something: to have and be able to use knowledge. This is increased in the trading zones of interdisciplinary or geographically fragmented work where social distance necessitates knowledge exchange without inter-personal trust. In these contexts, it is almost impossible to transfer somatic or collective tacit knowledge. Facing this, scientists seek workable levels of having 'enough' knowledge to get by, using simplifications, pidgin languages, and inter-personal face-to-face work to try to achieve a form of interactional expertise.

The importance of trust. Trust permeates knowledge exchange, but trust can take different forms. There is the issue of what is being trusted: a person, a software package, or a particular result encapsulated by the software. There is also the issue of what that trust is premised upon (inter-personal contact, institutional affiliation, confidence) and what forms of knowledge exchange this supports. Within the $4 \mathrm{Vs}$ (volume, variety, velocity and veracity) of big data, veracity or trustworthiness of data is becoming a key issue not only for long-tail scientists but for global social media in general (Zhou et al., 2014), where social distance exists in multiple directions between data producer and consumer.

Virtualisation and surveillance are two sides of the same coin. An unanticipated finding of our work was that ProtocolNavigator was understood by its users as a site of power through surveillance. As a software development team this was initially disappointing, as ProtocolNavigator had been associated with positive connotations of collaboration and data sharing, whereas surveillance is a negative connotation. However, the insight is not to see virtualisation and surveillance as two opposing forces in tension, but to recognise them as expressions of the same circumstance: both relate to visibility. ProtocolNavigator's virtual bench and linked interactive map provides a new form of visibility that can be simultaneously understood as empowering and disempowering in different ways (both the capacity to share connected activities through a virtual space and the capacity to be watched). Our investigation also showed how people's sense of self-identity and the identity of others are in part mediated by ProtocolNavigator. The collective surveillance aspects of virtualisation can be exploited to implement a collective governance structure, a key requirement for open-data sharing and good laboratory practice (Royal Society Science Policy Centre, 2012).

ProtocolNavigator did provoke learning, but through social means. The ProtocolNavigator trial was conducted under the challenging circumstances of an interdisciplinary team with geographical spread using in-development software to virtualise creative research practices. In this regard it is the toughest of settings to work in. Despite this we believe our case-study does evidence scientists gaining a better understanding of each other's work because of ProtocolNavigator. However, this improved understanding did not occur because users learned exclusively from the ProtocolNavigator output. Instead learning was part of a broader, situated, and practical learning experience, in which users were provoked into thinking about the collaborative work in new and deeper ways by the practice of interpreting the ProtocolNavigator output. In this way, the ProtocolNavigator output was a site of interpretative flexibility, and the users had to narrow down all of the possible interpretations. They did this by remembering what they heard in group presentations, or what they read elsewhere, or they emailed questions to other team members, or spoke in passing. ProtocolNavigator became one part of a bricolage of information that was assembled in interpreting the outputs. ProtocolNavigator did provoke learning, but as part of a broader set of practices that in part rely upon facework and immersion within the community.

Extended challenges of greater physical and social distance. The setting we studied saw high levels of social distance within the interdisciplinary team working through fractionated trading zones. However, the physical distance was low, with all participants experiencing levels of physical contiguity. As ProtocolNavigator-like systems seek to expand their reach in new contexts of application (e.g., geographically dispersed groups) the direct contact between team members may be reduced. In this context, the (prior or current) level of participation and physical contiguity within the included domains, but not with the specific individuals involved, could play an important role. As an example, an engineer working via ProtocolNavigator with experts in cell culture in another distant nation could attain some interactional expertise about cell culturing through physical contiguity with local cell culture experts, if the domain of cell culturing was sufficiently robust and common that unconnected contributory experts shared specialist tacit knowledge. This could support forms of institutional trust or trust-by-proxy (Reyes-Galindo, 2014), but cannot replace inter-personal trust, which would need to be developed through other mechanisms. Another aspect to be considered here would be the role of video demonstrations of cell culture practice embedded within ProtocolNavigator itself. These potentials may frame knowledge exchange, and the way trust relationships mediate it. In keeping with the broader conclusion of this study, at this stage we must only remark that understanding these possibilities is best achieved through empirical research embedded within a robust theoretical framework.

\section{Conclusion}

The work reported here is both an analysis of interdisciplinarity, and an example of interdisciplinarity. It is also a call for further work and an argument for the value of using interdisciplinary insight into guiding how it is conducted. We have shown how interdisciplinary work is situated and complex, and provided an expanded framework for understanding this. We have shown that ProtocolNavigator did aid the transfer of knowledge, although this was in part achieved through offline interactions provoked through ProtocolNavigator usage, and was mediated by the 
hierarchies and disciplinary cultural differences within the group. We believe by conducting this analysis we better illuminate the challenges within data science that supports interdisciplinarity, and the role of empirical social science in addressing them.

Data access. The data sets generated and analysed during the current study (interviews and fieldnotes) are not publicly available due to the confidentiality requirements agreed with participants. In the case of this data, even if made anonymous by name, the data may still be identifiable due to the specific and globally unique nature of the field site, so access is not available.

Received: 10 January 2018 Accepted: 30 April 2018

Published online: 26 June 2018

\section{References}

Alač M (2011) Handling digital brains: A laboratory study of multimodal semiotic interaction in the age of computers. MIT Press, Cambridge MA

Atkinson P.,Morriss L., (2017) On Ethnographic Knowledge Qualitative Inquiry 23 (5):323-331

Bartlett A, Lewis J, Reyes-Galindo L, Stephens N (2018) The locus of legitimate interpretation in Big Data sciences: Lessons for computational social science from -omic biology and high-energy physics. Big Data and Society https:// doi.org/10.1177/2053951718768831

Bauman Z, Lyon D (2013) Liquid surveillance: A conversation. Polity Press, Cambridge, England

Boyce T (2007a) Journalism and expertise. J Stud 7(6):889-906

Boyce T (2007b) Health, risk and news: the MMR vaccine and the media. Peter Lang, New York, NY

Bucher T (2012) Want to be on the top? Algorithmic power and the threat of invisibility on Facebook. New Media Soc 14(7):1164-1180

Burgun A, Bodenreider $\mathrm{O}(2008)$ Accessing and integrating data and knowledge for biomedical research Yearb Med Inform 17(1):91-101

Caluya G (2010) The post-panoptic society? Reassessing Foucault in surveillance studies. Social Identit- 16(5):621-633

Calvert J (2010) Systems biology, interdisciplinarity and disciplinary identity. In: Parker John N, Vermeulen Niki, Penders Bart (eds). Collaboration in the new life sciences. Ashgate, Farnham, pp. 201-219

Collins H (1992) Changing order: Replication and induction in scientific practice Chicago. Chicago University Press, Chicago

Collins H (2001) Tacit knowledge, trust, and the Q of sapphire. Soc Stud Sci 31 (1):71-85

Collins H (2007) Bicycling on the moon: Collective tacit knowledge and somaticlimit tacit knowledge. Organ Stud 28(2):257-262

Collins H (2010a) Tacit and explicit knowledge. University of Chicago Press, Chicago

Collins H (2010b) Tacit knowledge: you don't know how much you know. New Sci 206(2762):30-31

Collins H, Evans R (2002) The third wave of science studies: Studies of expertise and experience. Soc Stud Sci 32(2):235-296

Collins H, Evans R (2003) King Canute meets the beach boys: Responses to the third wave. Soc Stud Sci 33(3):435-452

Collins H, Evans R (2007) Rethinking Expertise. University of Chicago Press, Chicago

Collins H, Evans R (2015) Expertise Revisited, Part I-Interactional Expertise Stud Hist Philos Sci Part A 54:113-123

Collins H, Evans R (2016) A thousand words Is worth a picture Soc Stud Sci 46 (2):312-324

Collins H, Evans R (2017) The bearing of studies of expertise and experience on ethnography Qualitative Inquiry 23(6):445-451

Collins H, Evans R, Gorman M (2007) Trading zones and interactional expertise. Stud Hist Philos Sci 38(4):657-666

Collins H, Evans R, Weinel M (2017) STS as science or politics? Soc Stud Sci 47 (4):580-586

Collins H, Weinel M, Evans R (2010) The politics and policy of the third wave: New technologies and society. Crit Policy Stud 4(2):185-201

Collins H, Weinel M, Evans R (2011) Object and shadow: Responses to the CPS critiques of Collins, Weinel and Evans', 'politics and policy of the third wave'. Crit Policy Stud 5(3):340-348

Collins H, Evans R, Ribeiro R, Hall M (2006) Experiments with interactional expertise. Stud Hist Philos Sci 37(4):656-674
De Roure D, Goble C, Stevens R (2009) The design and realisation of the myExperiment Virtual Research Environment for social sharing of workflows. Future Gener Comput Syst 25(5):561-567

De Roure D, Goble C, Bhagat J et al. (2010) MyExperiment: a repository and social network for the sharing of bioinformatics workflows. Nucl Acids Res 38:182-189

Deleuze G, Guattari F (1987) A thousand plateaus. University of Minnesota Press, Minneapolis

Downes A, Mouras R, Bagnaninchi P et al. (2011) Raman spectroscopy and CARS microscopy of stem cells and their derivatives. Raman Spectrosc 42 (10): $1864-1870$

Edwards J, Thomas R, Guilliatt R (2017) Regenerative medicine: from the laboratory looking out. Palgrave Commun 3:27

Epstein S (2011) Misguided boundary work in studies of expertise: Time to return to the evidence. Crit Policy Stud 5(3):323-328

Felt U, Fouché R, Miller C, Smith-doerr L (2017) The handbook of science and technology studies, 4th edn. MIT Press, Cambridge, MA

Fischer F (2011) The 'policy turn' in the third wave: Return to the fact-value dichotomy? Crit Policy Stud 5(3):311-316

Forsyth T (2011) Expertise needs transparency not blind trust: A deliberative approach to integrating science and social participation. Crit Policy Stud 5 (3):317-322

Foucault M (1975) Discipline and punish: the Birth of the Prison. Random House, New York

Foucault M (1978) The history of sexuality vol. 1 an introduction. Allen Lane, London

Galison P (1996) Computer simulations and the trading zone. In: Galison P, Stump DJ (eds) The disunity of science: boundaries, contexts, and power. Stanford University Press, Stamford, pp. 118-157

Giddens A (1990) The consequences of modernity. Stanford University Press, Stanford

Giddens A (1991) Modernity and self-identity: Self and society in the late modern age. Stanford University Press, Stanford

Goddiksen M (2014) Clarifying interactional and contributory expertise Stud Hist Philos Sci Part A 47:111-117

Gorman M (2002) Levels of expertise and trading zones: A framework for multidisciplinary collaboration. Soc Stud Sci 32(5-6):933-938

Haggerty KD, Ericson RV (2000) The surveillant assemblage. Br J Sociol 51:605-622

Hartswood M, Procter R, Taylor P et al. (2012) Problems of data mobility and reuse in the provision of computer-based training for screening mammography. Proceedings of the 2012 Annual Conference on Human Factors in Computing Systems: ACM Conference on Human Factors in Computing Systems (CHI). ACM Press, Austin, TX, USA

Herbert D, Maschner G, Schou C (2013) Virtualization and the democratization of science. IEEE. 978-1-4799-3170-5/13.

Jasanoff S (2003) Breaking the waves in science studies: Comment on H.M. Collins and Robert Evans, 'The third wave of science studies'. Soc Stud Sci 33 (3):389-400

Jasanoff S, Markle G, Petersen J, Pinch T (1995) Handbook of science and technology studies, 2nd edn. Sage, Thousand Oaks

Jennings B (2011) Poets of the common good: Experts, citizens, public policy. Crit Policy Stud 5(3):334-339

Khan I, Fraser A, Bray M, Smith P, White N, Carpenter A, Errington R (2014) ProtocolNavigator: emulation-based software for the design, documentation and reproduction biological experiments. Bioinformatics 30(23):3440-3442

Klein J, Newell W (1998) Advancing Interdisciplinary Studies. In: Newell W (ed.) Interdisciplinarity: Essays from the literature. College Board, New York, pp. $3-22$

Knorr K (1977) Producing and reproducing knowledge: Descriptive or constructive? Toward a model of research production. Social Sci Inf 16:669-696

Latour B, Woolgar S (1985) Laboratory life: The construction of scientific facts. Princeton University Press, Princeton, NJ

Lewis J, Atkinson P (2011) The surveillance of cellular scientists' practice. BioSocieties 6(4):381-400

Lewis J, Bartlett A (2013) Inscribing a discipline: Tensions in the field of bioinformatics. New Genet Soc 32(3):243-263

Lewis J, Bartlett A, Atkinson P (2016) Hidden in the middle: culture, value and reward in bioinformatics. Minerva 54(4):471-490

Lu FK, Basu S, Igras V et al. (2015) Label-free DNA imaging in vivo with stimulated Raman scattering microscopy. Proc Natl Acad Sci USA 112 (37):11624-11629

Lupton D (2012) M-health and health promotion: The digital cyborg and surveillance society. Social Theory Health 10(3):229-244

Lynch M (1985) Art and artifact in laboratory science: A study of shop work and shoptalk in a research laboratory. Routledge \& Kegan Paul, London

MacKenzie D (2001) Mechanizing proof: Computing, risk, and trust. The MIT Press, Cambridge MA 
Morrison M (2017) A good collaboration is based on unique contributions from each side: assessing the dynamics of collaboration in stem cell science. Life Sci Soc Pol 13(7):1-20.

Owens S (2011) Three thoughts on the third wave. Crit Policy Stud 5(3):329-333

Penders B, Horstman K, VOs R (2008) Walking the line between lab and computation: The "Moist" zone. Bioscience 58:747-755

Plaisance K, Kennedy E (2014) A pluralistic approach to interactional expertise Stud Hist Philos Sci Part A 47:60-68

Reyes-Galindo L (2014) Linking the subcultures of physics: Virtual empiricism and the bonding role of trust. Soc Stud Sci 44(5):736-757

Reyes-Galindo L, Duarte T (2015) Bringing tacit knowledge back to contributory and interactional expertise: A reply to Goddiksen. Stud Hist Philos Sci Part A 49:99-102

Ribeiro R, Lima F (2016) The value of practice: A critique of interactional expertise Soc Stud Sci 46(2):312-324

Rip A (2003) Constructing expertise: In a third wave of science studies? Soc Stud Sci 33(3):419-434

Romano P, Giugno R, Pulvirenti A (2011) Tools and collaborative environments for bioinformatics research. Brief Bioinform 12(6):549-561

Royal Society Science Policy Centre (2012) Science as an open enterprise. The Royal Society Science Policy Centre report June 2012 DES24782 London, Royal Society

Sismondo S (2017) Casting a wider net: A reply to Collins, Evans and Weinel. Soc Stud Sci 47(4):587-592

Snowdon D, Churchill E, Munro A (2001) An introduction in collaborative virtual environments. Springer-Verlag, London

Stephens N, Atkinson P, Glasner P (2011) Documenting the doable and doing the documented: Bridging strategies at the UK Stem Cell Bank. Soc Stud Sci 41 (6):791-813

Stephens N, Lewis J (2017) Doing laboratory ethnography: reflections on method in scientific workplaces. Qual Res 17(2):202-216

Stephens N, Lewis J, Atkinson P (2013a) Closing the regulatory regress: GMP accreditation in stem cell laboratories. Sociol Health Illn 35(3):34560

Stephens P, Glen A, Masia F et al. (2013b) Nondestructive imaging of stem cells and their differentiated progeny. Wound Repair Regen 21(6):A84-A84

Traweek S (1988) Beamtimes and lifetimes: The world of high energy physicists. Harvard University Press, Cambridge, MA

van Nuenen T (2016) Playing the panopticon: Procedural surveillance in dark souls. Games Cult 11(5):510-527

Vertesi J (2015) Seeing like a Rover: How robots, teams and images craft knowledge of Mars. Chicago University Press, Chicago

Wallis JC, Rolando E, Borgman CL (2013) If we share data, will anyone use them? data sharing and reuse in the long tail of science and technology. PLoS ONE 8 (7):e67332
Weinel M (2007) Primary source knowledge and technical decision-making: Mbeki and the AZT debate. Stud Hist Philos Sci Part A 38(4):748-760

Witt $M$ (2008) Institutional repositories and research data curation in a distributed environment. Libr Trends 57(2):191-201

Wynne B (2003) Seasick on the third wave? subverting the hegemony of propositionalism: response to Collins \& Evans (2002). Soc Stud Sci 33(3):401-417

Zhou Z, Chawla N, Jin Y, Williams J (2014) Big data opportunities and challenges: Discussions from data analytics perspectives. IEEE Comput Intell Mag 9 (4):62-74

\section{Acknowledgements}

This work was supported by the EPSRC Research Council grant (Grant No. EP/H45848/ 1). We also thank our colleagues and participants in the study.

\section{Additional information}

Competing interests: The authors declare no competing interests.

Reprints and permission information is available online at http://www.nature.com/ reprints

Publisher's note: Springer Nature remains neutral with regard to jurisdictional claims in published maps and institutional affiliations.

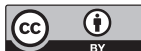

Open Access This article is licensed under a Creative Commons Attribution 4.0 International License, which permits use, sharing, adaptation, distribution and reproduction in any medium or format, as long as you give appropriate credit to the original author(s) and the source, provide a link to the Creative Commons license, and indicate if changes were made. The images or other third party material in this article are included in the article's Creative Commons license, unless indicated otherwise in a credit line to the material. If material is not included in the article's Creative Commons license and your intended use is not permitted by statutory regulation or exceeds the permitted use, you will need to obtain permission directly from the copyright holder. To view a copy of this license, visit http://creativecommons.org/ licenses/by/4.0/

(C) The Author(s) 2018 